\title{
Underdiagnosis of Dementia: an Observational Study of Patterns in Diagnosis and Awareness in US Older Adults
}

\author{
Halima Amjad, MD, MPH ${ }^{1,2}$, David L. Roth, $P h D^{1,2}$, Orla C. Sheehan, $M D, P h D^{1,2}$, \\ Constantine G. Lyketsos, MD, MHS ${ }^{3}$, Jennifer L. Wolff, PhD ${ }^{2,4}$, and Quincy M. Samus, $P h D^{2,3}$
}

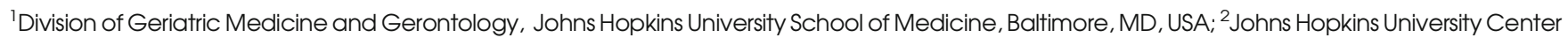
on Aging and Health, Baltimore, MD, USA; ${ }^{3}$ Department of Psychiatry and Behavioral Sciences, Johns Hopkins University School of Medicine, Baltimore, MD, USA; ${ }^{4}$ Department of Health Policy and Management, Johns Hopkins University Bloomberg School of Public Health, Baltimore, MD, USA.

BACKGROUND: Many older adults living with dementia have not been formally diagnosed. Even when clinicians document the diagnosis, patients and families may be unaware of the diagnosis. Knowledge of how individual characteristics affect detection and awareness of dementia is limited.

OBJECTIVE: To identify characteristics associated with dementia diagnosis and awareness of diagnosis.

DESIGN: Cross-sectional observational study.

PARTICIPANTS: Five hundred eighty-five adults aged $\geq$ 65 in the National Health and Aging Trends Study who met assessment-based study criteria for probable dementia in 2011 and had 3 years of continuous, fee-for-service Medicare claims prior to 2011.

MAIN MEASURES: Using multivariable logistic regression, we compared participants with undiagnosed versus diagnosed dementia (based on Medicare claims) on demographic, social/behavioral, functional, medical, and healthcare utilization characteristics. Among those diagnosed, we compared characteristics of participants unaware versus aware of the diagnosis (based on self or proxy report).

KEY RESULTS: Among older adults with probable dementia, $58.7 \%$ were either undiagnosed (39.5\%) or unaware of the diagnosis (19.2\%). In adjusted analyses, individuals who were Hispanic (OR 2.48, 95\% CI 1.19, 5.14), had less than high school education (OR 0.54 for at least high school education, 95\% CI 0.32, 0.91), attended medical visits alone (OR 1.98, 95\% CI 1.11, 3.51), or had fewer functional impairments (OR 0.79 for each impairment, 95\% CI 0.69, 0.90) were more likely to be undiagnosed. Similarly, among those diagnosed, having less education (OR 0.42), attending medical visits alone (OR 1.97), and fewer functional impairments (OR 0.72 ) were associated with unawareness of diagnosis (all ps < 0.05).

CONCLUSIONS: The majority of older adults with dementia are either undiagnosed or unaware of the diagnosis, suggesting shortcomings in detection and communication of dementia. Individuals who may benefit from targeted screening include racial/ethnic minorities and persons who have lower educational attainment, any functional impairment, or attend medical visits alone.

Received August 30, 2017

Revised December 28, 2017

Accepted February 8, 2018

Published online March 5, 2018
KEYWORDS: Geriatrics; Dementia; Alzheimer's; Diagnosis; Vulnerable populations.

J Gen Intern Med 33(7):1131-8

DOI: $10.1007 / \mathrm{s} 11606-018-4377-\mathrm{y}$

(c) Society of General Internal Medicine 2018

\section{INTRODUCTION}

Approximately 5.5 million people in the USA are living with dementia ${ }^{1}$. Despite high prevalence and important implications for patients and families, dementia is underdiagnosed by clinicians and underreported by patients and families. Only about half of individuals who meet the criteria for dementia are diagnosed by a clinician ${ }^{2-6}$.

Timely diagnosis of dementia may be important in improving patient outcomes both now and when effective treatments become available. Even in the absence of disease-modifying treatment, early diagnosis of dementia may have implications for mitigating safety risks ${ }^{6}$, prevention of complications, and patient/family planning for the future ${ }^{7}$. Recent guidelines, however, found insufficient evidence to support universal screening for dementia ${ }^{8}$, and results of the only RCT of benefits and harms of screening are pending (IU-CHOICE Study, NCT01699503) ${ }^{9}$. Uncovering links between timely dementia diagnosis and improved outcomes requires better understanding the scope of the problem and characteristics of the population living with undiagnosed dementia. Unique characteristics of undiagnosed individuals could be translated into targeted dementia detection, education, and support.

Studies have begun to build sociodemographic and medical profiles of individuals living with undiagnosed dementia ${ }^{2-5}$, 10-12. However, methodology has been limited in important ways. Lack of awareness of a diagnosis, based on patient/ proxy report of clinician diagnosis, has been used as a surrogate for undiagnosed dementia ${ }^{3,13}$. Conversely, documentation of diagnosis by providers in medical records $4,5,10-12$ does not necessarily translate into patient or family awareness of the diagnosis. Examining either reported diagnosis or documentation provides an incomplete picture of dementia diagnosis and awareness; both elements are necessary for patients and families to understand patient cognitive and functional 
limitations and prognosis. No studies have examined documented dementia diagnosis together with patient or family awareness of a dementia diagnosis. In addition, few studies 3,13 have examined nationally representative populations.

To address this knowledge gap, we draw on a nationally representative study to advance understanding of who has undiagnosed dementia and who has diagnosed dementia but is not aware of the diagnosis. We compare demographic, social/behavioral, functional, medical, and healthcare utilization characteristics of older adults with dementia by both documented diagnosis in claims records (undiagnosed versus diagnosed) and patient/family awareness of the condition (unaware versus aware among the diagnosed) in the National Health and Aging Trends Study (NHATS).

\section{METHODS}

\section{Study Design and Participants}

We examined baseline data from NHATS in 2011 linked to retrospective Medicare A and B claims data. NHATS follows a population-based, nationally representative cohort of Medicare beneficiaries aged $\geq 65$ in the continental USA. Participants or proxy respondents undergo annual interviews assessing sociodemographics, functional status, health status, and medical care among other topics. Proxies were used when a participant could not fully respond due to health or memory problems; preference was given to someone who lived with the participant or was familiar with participant health and routine ${ }^{14}$.

Figure 1 displays the process of obtaining our analytic sample of 585 older adults with probable dementia. NHATS enrolled 8245 participants in 2011 (71\% overall response rate) ${ }^{15}$.

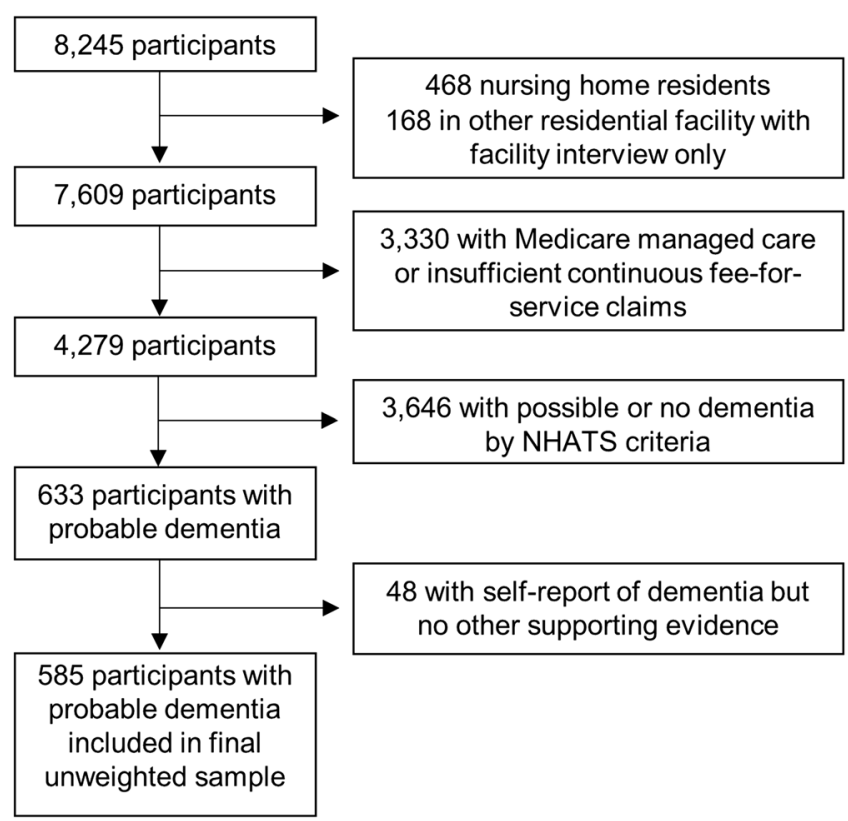

Figure 1 Flow of participants to obtain final analytic sample from the National Health and Aging Trends Study (NHATS).
We included participants who were community-dwelling ( $n=$ 636 excluded who lived in a residential facility) and continuously enrolled in fee-for-service Medicare over the prior 3 years ( $n=3330$ excluded). We then narrowed our sample to include only participants with probable dementia.

NHATS investigators have developed and validated an algorithm to identify dementia status (i.e., probable dementia vs. no dementia) ${ }^{16}$. Probable dementia is defined by meeting at least one of three criteria: self or proxy report of clinician diagnosis of dementia or Alzheimer's disease, score $\geq 2$ on the AD-8 Dementia Screening Interview administered to proxies ${ }^{17}$, or score $>1.5$ standard deviations below the mean for selfrespondents in two or three cognitive domains tested $(n=434$ in our sample completed cognitive testing). The AD- 8 Dementia Screening Interview is an eight-item questionnaire validated for classifying dementia by informant interview ${ }^{17}$, ${ }^{18}$. Cognitive tests assessed memory (immediate and delayed 10-word recall), orientation (day, date, month, year, President and Vice President), and executive function (clock drawing test). The NHATS dementia classification algorithm resulted in 633 persons with probable dementia based on report of diagnosis, proxy screening interview, and/or cognitive testing ( $n=3646$ excluded).

\section{Dementia Diagnosis and Awareness}

The outcomes of interest were diagnosis of dementia and awareness of the diagnosis. Participants with probable dementia were thus categorized by whether they had a Medicare claims diagnosis of dementia and if diagnosed, by whether they or their proxy was aware of the diagnosis. Claims-based dementia diagnosis was determined using the Medicare Chronic Conditions Warehouse (CCW) indicator for Alzheimer's disease and related disorders or senile dementia. The $\mathrm{CCW}$ indicator requires that beneficiaries have at least one inpatient, skilled nursing facility, home health, Part B institutional, or Part B non-institutional claim with any of 24 ICD-9 dementia codes over a 3-year reference period ${ }^{19}$. Awareness of diagnosis was based on self or proxy report of a clinician diagnosis of dementia or Alzheimer's disease in NHATS. We excluded 48 participants who met NHATS criteria for probable dementia based on self or proxy report of diagnosis but lacked a corresponding claims diagnosis. Dementia status was questionable in this group as 35 of these 48 participants did not have any other objective supporting data (cognitive testing or proxy interview) for dementia. The resulting three groups $(n=585)$ examined were undiagnosed $(n=229)$, diagnosed but unaware of diagnosis $(n=116)$, or diagnosed and aware of diagnosis $(n=240)$.

\section{Predictors of Diagnosis and Awareness}

The three dementia groups were compared on demographic, social and behavioral, functional status, and medical and healthcare utilization factors. 
Demographic Factors. From NHATS, we included age, gender, and self-identified race/ethnicity (non-Hispanic White, non-Hispanic Black, and Hispanic or other non-Hispanic race [Asian, Pacific Islander, Native American]).

Social and Behavioral Factors. We examined marital status (married/living with partner versus single/widowed/ divorced), number of children, and geographic location (based on census division reported in NHATS). Socioeconomic status (SES) components included highest level of education (less than high school, high school degree and/or some college/trade school, Associate's degree or greater) and total annual income. We used imputed total income provided by NHATS for participants without reported income ${ }^{20}$. Medicare-Medicaid dual eligibility status was derived from the Medicare enrollment file. Living alone and going to doctor visits alone were also included.

Functional Status Factors. Activity of daily living (ADL) impairments were assessed through reported difficulty or help required for bathing, eating, dressing, and toileting. Instrumental activity of daily living (IADL) impairments were assessed through reported difficulty or help required in cooking, managing finances, managing medications, shopping, and doing laundry ${ }^{21}$. We examined mean ADL and IADL impairments, individual activity impairments, and mobility impairment (not being able to go outside without help).

Medical and Healthcare Utilization Factors. From NHATS, we examined self-reported falls in the past year. Depression and anxiety in NHATS were assessed through $\mathrm{Pa}$ tient Health Questionnaire-2 and Generalized Anxiety Disorder-2 scales, respectively, with scores $>2$ considered positive ${ }^{22,23}$. We measured multimorbidity using a modified claims-based Charlson Comorbidity Index that excluded dementia ${ }^{24}$. We measured any hospitalization in the past year through Medicare Part A claims and any emergency room (ER) visit and mean ambulatory visits in the past year through Part B outpatient claims and the Carrier file. We derived whether participants saw a primary care provider (internal, family, general, or geriatric medicine physician; nurse practitioner) in the past year using provider specialty codes in outpatient claims.

Additional Covariates. We examined characteristics that may affect the relationship between predictors and dementia diagnosis and awareness. We accounted for type of respondent (proxy, self-respondent with assistance, or independent self-respondent) as respondent type may affect accuracy of responses. To better account for dementia severity, we also considered cognitive test scores for participants who completed testing.

\section{Statistical Analysis}

Analytic sampling weights that account for non-response bias and the complex sampling strategy were applied in all analyses ${ }^{25}$. We first used simple inferential statistics (chi-square, $t$ test, or ANOVA) to compare characteristics between groups with probable dementia (undiagnosed, diagnosed but unaware, and diagnosed and aware).

We then constructed two sets of logistic regression models. The first set of models compared persons with undiagnosed dementia to those with diagnosed dementia (the reference group). The second set focused on the diagnosed dementia sub-sample and compared persons unaware to those aware of the dementia diagnosis (the reference group). The same set of predictors was included in each model. To build final multivariable regression models, we sequentially evaluated variables by category, including demographic covariates and each category of characteristics. Thus, these models examined diagnosis and then awareness with the following categories of covariates: demographics only, demographics and social and behavioral factors, demographics and mean ADL and IADL impairments, demographics and medical/healthcare utilization factors, and demographics and individual functional activity impairments.

We selected individual variables for inclusion in the final multivariable logistic regression models based on statistical significance $(p<0.05)$ in the category-level regression models or a priori planned inclusion (age, gender, education, depression, anxiety). Depression and anxiety were included as neuropsychiatric symptoms are often presenting symptoms of dementia ${ }^{26}$. Respondent type was included in sensitivity analysis. Subgroup analysis added cognitive test scores to final models as the entire sample did not complete these items.

Analyses were conducted using Stata v14 (StataCorp LP; College Station, TX). The Johns Hopkins University (JHU) Bloomberg School of Public Health Institutional Review Board (IRB) oversees NHATS; this study was approved by the JHU School of Medicine IRB.

\section{RESULTS}

The 585 participants who met criteria for probable dementia and had fee-for-service Medicare yielded a weighted population estimate of 1,972,534 individuals. Dementia was undiagnosed in $39.5 \%$ of individuals. Among diagnosed persons, $31.8 \%$ of those persons or their proxies were unaware of the diagnosis.

Table 1 displays participant characteristics in the three groups. There were no statistically significant differences in marital status, living alone, geographic location, Charlson Comorbidity Index, ER or mean ambulatory visits in the past year, depression, or anxiety across groups. Overall, most proxy respondents were either a daughter (33.2\%), spouse/partner (28.0\%), or son $(16.1 \%)$; at least $87 \%$ of proxies in each group reported being very familiar with the participant's routine. 
Table 1 Characteristics of the Weighted NHATS Sample with Probable Dementia and Fee-For-Service Medicare

\begin{tabular}{|c|c|c|c|c|c|}
\hline & \multicolumn{3}{|c|}{ Dementia status } & \multicolumn{2}{|l|}{$P$ value* } \\
\hline & Undiagnosed & $\begin{array}{l}\text { Diagnosed } \\
\text { but unaware }\end{array}$ & $\begin{array}{l}\text { Diagnosed } \\
\text { and aware }\end{array}$ & 3 groups & $\begin{array}{l}2 \text { diagnosed } \\
\text { groups }\end{array}$ \\
\hline Weighted $\% \dagger$ & 39.5 & 19.2 & 41.3 & - & - \\
\hline \multicolumn{6}{|l|}{ Demographic factors } \\
\hline Age, years, mean (SE) & $80.9(0.6)$ & $83.6(0.7)$ & $83.2(0.4)$ & 0.02 & 0.65 \\
\hline Female, $\%$ & 55.8 & 65.4 & 70.6 & 0.03 & 0.45 \\
\hline \multicolumn{6}{|l|}{ Race/ethnicity, \% } \\
\hline White, non-Hispanic & 63.7 & 82.7 & 78.2 & $<0.001$ & 0.41 \\
\hline Black, non-Hispanic & 12.5 & 10.3 & 11.2 & & \\
\hline Hispanic or other non-Hispanic & 21.4 & 6.7 & 9.1 & & \\
\hline \multicolumn{6}{|l|}{ Social and behavioral factors } \\
\hline Married/living with partner, \% & 37.0 & 32.9 & 38.7 & 0.61 & 0.51 \\
\hline Living children, mean (SE) & $3.2(0.1)$ & $2.5(0.2)$ & $2.9(0.1)$ & 0.05 & 0.10 \\
\hline Lives alone, $\%$ & 31.4 & 45.8 & 33.4 & 0.06 & 0.06 \\
\hline \multicolumn{6}{|l|}{ Highest education, $\%$} \\
\hline$<$ High school & 49.3 & 40.9 & 30.2 & 0.01 & 0.22 \\
\hline$\geq$ High school, no higher degree & 34.8 & 43.6 & 45.9 & & \\
\hline$\geq$ Associate's degree & 12.4 & 13.7 & 20.9 & & \\
\hline Annual income, $\$$, mean (SE) & $22,932(2625)$ & $23,415(3083)$ & $41,699(8110)$ & 0.06 & $<0.001$ \\
\hline Dual eligible, $\%$ & 37.0 & 25.9 & 20.4 & 0.002 & 0.23 \\
\hline Went to doctor alone, $\%$ & 27.1 & 17.3 & 7.1 & $<0.001$ & 0.009 \\
\hline \multicolumn{6}{|l|}{ Functional status factors } \\
\hline IADL impairments, mean (SE) & $1.9(0.1)$ & $2.4(0.1)$ & $3.3(0.1)$ & $<0.001$ & $<0.001$ \\
\hline ADL impairments, mean (SE) & $2.2(0.1)$ & $2.1(0.1)$ & $3.5(0.1)$ & $<0.001$ & $<0.001$ \\
\hline \multicolumn{6}{|l|}{ Specific IADL impairment, \% } \\
\hline Laundry & 38.1 & 35.9 & 53.4 & 0.005 & 0.01 \\
\hline Shopping & 48.2 & 56.3 & 73.6 & $<0.001$ & 0.001 \\
\hline Cooking & 40.5 & 43.6 & 57.5 & 0.003 & 0.05 \\
\hline Finances & 36.0 & 61.5 & 81.8 & $<0.001$ & 0.002 \\
\hline Medication management & 36.0 & 44.5 & 70.9 & $<0.001$ & $<0.001$ \\
\hline Outside mobility & 48.8 & 41.8 & 57.3 & 0.02 & 0.01 \\
\hline Drove in past month, $\%$ & 27.8 & 22.6 & 13.8 & 0.002 & 0.06 \\
\hline \multicolumn{6}{|l|}{ Medical and health utilization factors } \\
\hline Charlson Index, mean (SE) $\ddagger$ & $2.4(0.1)$ & $2.8(0.3)$ & $2.5(0.1)$ & 0.49 & 0.30 \\
\hline Hospitalized in past year, $\%$ & 23.5 & 40.6 & 39.4 & $<0.001$ & 0.80 \\
\hline ER visit in past year, \% & 33.6 & 46.6 & 37.5 & 0.11 & 0.14 \\
\hline Ambulatory visits in past year, mean (SE) & $8.3(0.4)$ & $10.0(0.9)$ & $8.9(0.5)$ & 0.44 & 0.32 \\
\hline Primary care provider seen in past year, $\%$ & 83.8 & 93.5 & 94.7 & 0.002 & 0.70 \\
\hline Fall in past year, $\%$ & 45.4 & 36.8 & 54.7 & 0.01 & 0.002 \\
\hline Depression, $\% \S$ & 33.6 & 31.3 & 39.7 & 0.34 & 0.23 \\
\hline Anxiety, \% & 27.4 & 26.2 & 24.2 & 0.73 & 0.69 \\
\hline \multicolumn{6}{|l|}{ Cognitive test scores $\mathbb{I}$} \\
\hline Orientation, mean (SE) & $3.3(0.1)$ & $2.0(0.1)$ & $2.3(0.2)$ & $<0.001$ & 0.08 \\
\hline Memory, mean (SE) & $1.8(0.1)$ & $1.5(0.1)$ & $2.5(0.2)$ & 0.003 & $<0.001$ \\
\hline Executive function, mean (SE) & $1.3(0.1)$ & $1.6(0.1)$ & $1.6(0.1)$ & 0.09 & 0.63 \\
\hline \multicolumn{6}{|l|}{ Respondent type } \\
\hline Proxy respondent, \% & 37.3 & 28.7 & 55.9 & $<0.001$ & 0.002 \\
\hline Self with assistant, \% & 42.5 & 32.9 & 41.3 & 0.23 & 0.14 \\
\hline Self, \% & 20.2 & 38.4 & 2.8 & $<0.001$ & $<0.001$ \\
\hline
\end{tabular}

NHATS National Health and Aging Trends Study, SE standard error, IADL instrumental activities of daily living, ADL activities of daily living, ER emergency room

Determined by chi-square, $t$ test, or ANOVA test statistics

${ }^{\prime}$ Unweighted $n=585$

${ }^{*}$ Charlson comorbidity index calculation excluded dementia

${ }^{8} P H Q-2$ score $>2$

"GAD-2 score $>2$

"470 participants in unweighted sample completed cognitive test items. Score range 0-8 for orientation, 0-20 for memory, and 0-5 for executive function; higher scores are better

Unadjusted comparisons indicated that persons with undiagnosed dementia were younger and more likely to be male and non-White compared to diagnosed persons. Undiagnosed persons were more likely to be dually eligible, have less than high school education, and attend doctor visits alone. Functionally, this group had fewer IADL impairments compared to those diagnosed. With regard to healthcare utilization, persons with undiagnosed dementia were less likely to have been hospitalized or evaluated in the ER in the past year. Undiagnosed individuals were less likely to have seen a primary care provider in the prior year but had similar rates of ambulatory visits, comorbidity burden, depression, and anxiety.

Comparing self or family awareness of diagnosis among persons with diagnosed dementia, older adults unaware of the diagnosis had lower mean income, fewer IADL and ADL impairments, and were more likely to go to doctor visits alone compared to those aware of the diagnosis. Persons unaware of the diagnosis were less likely to have a proxy respondent. 


\section{Undiagnosed Versus Diagnosed Dementia}

In multivariable adjusted analyses (Table 2), differences in diagnosis persisted in race, education, IADL impairments, and going to doctor visits alone. Non-White race consistently demonstrated higher odds of undiagnosed versus diagnosed dementia, though the OR was statistically significant only for Hispanic/other non-White race participants. Individuals with at least high school education had $46 \%$ lower odds of being undiagnosed compared to individuals with lower education levels $(p=0.02)$. Individuals who attended doctor visits alone had almost twice the odds of being undiagnosed compared to individuals accompanied to doctor visits. IADL impairments were positively associated with dementia diagnosis. In sensitivity and subgroup analyses, the statistically significant associations of going to doctor visits alone and IADL impairments persisted; the associations of race and education varied in significance though magnitude of the ORs remained similar.

When IADL impairments were examined individually, persons with impairment in managing finances had $86 \%$ lower risk of undiagnosed dementia (adjusted OR 0.14, 95\% CI 0.07, 0.26 ), and persons with impairment in managing medications had $69 \%$ lower risk (adjusted OR 0.31, 95\% CI 0.18, 0.53).

\section{Unaware Versus Aware of Dementia Diagnosis}

In multivariable adjusted analyses (Table 3), there were statistically significant associations of education and IADL impairments with diagnosis awareness. Persons with at least an associate's degree had higher odds of being aware of their diagnosis. Similarly, persons with greater IADL impairments also had higher odds of awareness. Going to the doctor alone was associated with lower odds of being aware of the dementia diagnosis after adjusting for cognitive test scores. Self- respondents had much higher odds of not being aware of their dementia diagnosis compared to proxies. When IADL impairments were examined individually, impairment in managing medications was associated with lower odds of being unaware of diagnosis (adjusted OR 0.30, 95\% CI 0.12, 0.75).

\section{DISCUSSION}

This investigation evaluated diagnosis and awareness of dementia in a nationally representative cohort. Only $41 \%$ of older adults with probable dementia are both diagnosed and aware of the diagnosis. Undiagnosed individuals are more likely to be non-White and have lower educational attainment and less functional impairment. These individuals are also more likely to attend doctor visits without a companion. Among persons diagnosed, individuals unaware of the diagnosis are also more likely to have lower educational attainment and less functional impairment and to attend doctor visits alone. Impairments in managing finances and medications portend greater likelihood of dementia diagnosis and patient/ family awareness compared to other IADL impairments. These characteristics can guide improved dementia detection and communication in clinical practice.

The low likelihood of detection and awareness found in this study is similar to previous estimates based on either documented or reported diagnosis ${ }^{3-5,10-12}$. Several studies have found that older age ${ }^{3,10,11}$ and male gender ${ }^{2,3}$ are associated with undetected dementia. After adjusting for other factors, we found no association with age or gender. The positive association between functional impairment, diagnosis, and awareness is consistent with prior studies ${ }^{3,10,11,13}$.

Table 2 Odds Ratio and 95\% CI for Undiagnosed Versus Diagnosed Dementia among the Weighted NHATS Sample with Fee-For-Service Medicare

\begin{tabular}{|c|c|c|c|c|}
\hline Variable & Unadjusted & Multivariable adjusted & Respondent adjusted & Cognitive test subgroup \\
\hline Age & $0.96 \dagger(0.93,0.98)$ & $0.98(0.95,1.02)$ & $0.98(0.95,1.02)$ & $0.99(0.95,1.03)$ \\
\hline Gender $(\mathrm{ref}=$ male $)$ & $0.60 *(0.39,0.94)$ & $0.82(0.48,1.39)$ & $0.83(0.49,1.41)$ & $0.89(0.47,1.68)$ \\
\hline \multicolumn{5}{|l|}{ Race (ref = white) } \\
\hline Black & $1.35(0.95,1.92)$ & $1.26(0.82,1.95)$ & $1.26(0.81,1.97)$ & $1.28(0.76,2.17)$ \\
\hline Hispanic/other & $3.32 \dagger(1.71,6.45)$ & $2.48 *(1.19,5.14)$ & $2.44 *(1.19,5.00)$ & $2.37(0.97,5.83)$ \\
\hline \multicolumn{5}{|l|}{ Education $(\mathrm{ref}=<\mathrm{HS})$} \\
\hline$\geq \mathrm{HS}$, no higher degree & $0.55+(0.36,0.83)$ & $0.54 *(0.32,0.91)$ & $0.54 *(0.32,0.92)$ & $0.66(0.38,1.14)$ \\
\hline$\geq$ Associate's degree & $0.50(0.25,1.01)$ & $0.52(0.25,1.12)$ & $0.52(0.25,1.11)$ & $0.59(0.22,1.57)$ \\
\hline Went to doctor's alone $(\mathrm{ref}=\mathrm{no})$ & $3.15+(1.81,5.46)$ & $1.98 *(1.11,3.51)$ & $2.04 *(1.11,3.75)$ & $1.94 *(1.06,3.55)$ \\
\hline Depression $(\mathrm{ref}=\mathrm{no})$ & $0.85(0.57,1.25)$ & $0.76(0.47,1.23)$ & $0.76(0.47,1.22)$ & $0.84(0.44,1.61)$ \\
\hline Anxiety $(\mathrm{ref}=\mathrm{no})$ & $1.14(0.78,1.68)$ & $1.40(0.87,2.26)$ & $1.40(0.87,2.26)$ & $1.50(0.75,2.99)$ \\
\hline Hospitalized in past year $(\mathrm{ref}=\mathrm{no})$ & $0.48+(0.30,0.75)$ & $0.76(0.44,1.31)$ & $0.76(0.44,1.31)$ & $0.61(0.32,1.16)$ \\
\hline PCP seen in past year $(r e f=n o)$ & $0.34 \dagger(0.17,0.68)$ & $0.52(0.25,1.08)$ & $0.51(0.24,1.09)$ & $0.57(0.26,1.24)$ \\
\hline Total IADL impairments & $0.74+(0.67,0.82)$ & $0.79 \dagger(0.69,0.90)$ & $0.79 \dagger(0.69,0.90)$ & $0.80 \div(0.68,0.94)$ \\
\hline \multicolumn{5}{|l|}{ Respondent type (ref = proxy) } \\
\hline Self & $1.78(0.92,3.46)$ & - & $0.94(0.43,2.05)$ & - \\
\hline Self with assistant & $1.45(0.89,2.36)$ & - & $1.07(0.61,1.87)$ & - \\
\hline \multicolumn{5}{|l|}{ Cognitive test scores } \\
\hline Orientation & $1.29 \dagger(1.18,1.42)$ & - & - & $1.32 \dagger(1.17,1.49)$ \\
\hline Memory & $0.96(0.88,1.05)$ & - & - & $0.88 *(0.78,0.99)$ \\
\hline Executive function & $0.86 *(0.74,0.99)$ & - & - & $0.82 *(0.68,0.99)$ \\
\hline
\end{tabular}

NHATS National Health and Aging Trends Study, HS high school, IADL instrumental activities of daily living, PCP primary care provider ${ }^{*} p<0.05$ $p<0.01$ 
Table 3 Odds Ratio and 95\% CI for Unaware Versus Aware of Dementia Diagnosis among the Weighted NHATS Sample with Diagnosed Dementia and Fee-For-Service Medicare

\begin{tabular}{|c|c|c|c|c|}
\hline Variable & Unadjusted & Multivariable adjusted & Respondent adjusted & $\overline{\text { Cognitive test subgroup }}$ \\
\hline Age & $1.01(0.97,1.06)$ & $1.02(0.96,1.07)$ & $1.03(0.97,1.09)$ & $0.99(0.93,1.07)$ \\
\hline Gender $($ ref = male $)$ & $0.90(0.42,1.92)$ & $0.81(0.36,1.81)$ & $0.63(0.27,1.44)$ & $0.78(0.33,1.86)$ \\
\hline \multicolumn{5}{|l|}{ Race (ref = white) } \\
\hline Black race & $0.78(0.44,1.38)$ & $0.73(0.35,1.51)$ & $0.76(0.36,1.62)$ & $0.70(0.31,1.54)$ \\
\hline Hispanic/other race & $0.65(0.21,2.04)$ & $0.87(0.25,3.09)$ & $0.84(0.20,3.47)$ & $0.66(0.12,3.60)$ \\
\hline \multicolumn{5}{|l|}{ Education $($ ref $=<\mathrm{HS})$} \\
\hline$\geq \mathrm{HS}$, no higher degree & $0.66(0.32,1.35)$ & $0.42 *(0.19,0.94)$ & $0.45(0.18,1.12)$ & $0.48(0.21,1.08)$ \\
\hline$\geq$ Associate's degree & $0.41 *(0.17,0.99)$ & $0.23 \dagger(0.09,0.61)$ & $0.29 *(0.10,0.81)$ & $0.30(0.09,1.04)$ \\
\hline Went to doctor's alone $($ ref $=$ no) & $2.45^{*}(1.10,5.48)$ & $1.97(0.77,5.02)$ & $1.75(0.66,4.68)$ & $5.23 \dagger(1.90,14.38)$ \\
\hline Depression $(\mathrm{ref}=\mathrm{no})$ & $0.65(0.33,1.29)$ & $0.69(0.23,2.03)$ & $0.85(0.29,2.52)$ & $0.94(0.34,2.61)$ \\
\hline Anxiety $($ ref = no) & $1.06(0.57,1.97)$ & $1.18(0.40,3.45)$ & $1.36(0.41,4.54)$ & $1.23(0.39,3.87)$ \\
\hline Hospitalized in past year $(\mathrm{ref}=\mathrm{no})$ & $0.99(0.64,1.53)$ & $1.08(0.59,1.97)$ & $0.87(0.49,1.55)$ & $0.89(0.45,1.77)$ \\
\hline PCP seen in past year $(\mathrm{ref}=\mathrm{no})$ & $1.27(0.36,4.45)$ & $1.23(0.25,6.16)$ & $1.58(0.27,9.28)$ & $0.97(0.17,5.49)$ \\
\hline Total IADL impairments & $0.74 \dagger(0.63,0.87)$ & $0.72 \dagger(0.59,0.88)$ & $0.82 *(0.68,0.99)$ & $0.73 *(0.57,0.94)$ \\
\hline \multicolumn{5}{|l|}{ Respondent type (ref = proxy) } \\
\hline Self & $8.77+(3.10,24.79)$ & - & $5.56 \dagger(2.02,21.23)$ & - \\
\hline Self with assistant & $1.62(0.70,3.78)$ & - & $1.16(0.46,2.92)$ & - \\
\hline \multicolumn{5}{|l|}{ Cognitive test scores } \\
\hline Orientation & $0.92(0.80,1.05)$ & - & - & $0.99(0.79,1.24)$ \\
\hline Memory & $0.84 \dagger(0.77,0.92)$ & - & - & $0.76 \dagger(0.66,0.87)$ \\
\hline Executive function & $0.95(0.77,1.16)$ & - & - & $1.04(0.82,1.33)$ \\
\hline
\end{tabular}

NHATS National Health and Aging Trends Study, HS high school, IADL instrumental activities of daily living, PCP primary care provider ${ }^{*} p<0.05$

$t_{p}<0.01$

Race and SES findings have been mixed 3, 10, 27, 28; we found educational attainment to be a significant factor. NonWhite older adults were more likely to be undiagnosed. Interestingly, once diagnosed, there was a trend toward greater awareness in non-White individuals. Prior studies also suggest that diagnosis is delayed in minority older adults ${ }^{29}$ despite higher dementia incidence in non-White populations ${ }^{30,} 31$. Disparities in diagnosis may be due to differences in knowledge and misconceptions that dementia represents normal aging 32 , ${ }^{33}$, clinician failure to recognize dementia ${ }^{34}$, cultural bias in cognitive assessment instruments ${ }^{35}$, and/or racial and ethnic differences in family support and expectations in function. Posthoc examination of select characteristics by race demonstrates that while proxy use was similar, Hispanic/other non-White race participants were most likely to have an interview assistant and live with others. These differences suggest greater family support though they were also more likely to go to doctor visits alone. The latter difference could reflect beliefs that cognitive symptoms represent normal aging in the absence of a dementia diagnosis. Further research into racial/ethnic differences is warranted to understand and address disparities.

Several findings are notable. First, going to doctor visits alone, a novel risk factor, was associated with greater likelihood of being undiagnosed and unaware of a dementia diagnosis. As the magnitude of association diminished after adjusting for cognitive test scores, these individuals may have relatively higher levels of cognitive function that may mask deficits, rendering diagnosis more difficult. Awareness in this group may be affected by poor insight and memory. Type of respondent (proxy versus self) was also strongly associated with awareness, further underscoring importance of having a knowledgeable informant to dementia diagnosis and awareness. Second, hospitalization was not associated with diagnosis or awareness. Improving rates of diagnosis and communication of the diagnosis may therefore not increase burden on inpatient care systems. Furthermore, there are individuals receiving inpatient care in which dementia remains undetected despite health system interactions. Lastly, though neuropsychiatric symptoms are common in dementia, symptoms of depression and anxiety do not significantly affect likelihood of diagnosis and awareness.

There are several limitations in this study. First, while most participants had a proxy or assistant, a sizable minority of undiagnosed or unaware participants responded independently; self-report may limit accuracy of responses in dementia. While sensitivity analyses considered respondent type, dementia awareness may differ if family members were interviewed. The fact that individuals with diagnosed dementia independently completed a lengthy research interview, however, suggests that their families may also be unaware of the diagnosis. Second, while there are strengths to using a population-based, nationally representative survey, probable dementia criteria were epidemiologic rather than clinical. NHATS dementia classification has been shown to have acceptable sensitivity and specificity (66 and $87 \%$, respectively) 16 . Including only individuals with sufficient Medicare fee-forservice claims to ascertain dementia diagnosis limits generalizability to Medicare-managed care enrollees. In addition, there may be discrepancies between medical record documentation and billing codes; however, requiring only one claim for dementia over 3 years makes missed diagnoses less likely. Finally, findings might differ if the data were obtained today rather than 2011.

This study has clinical practice implications given gaps in detection of dementia and communication of the diagnosis to patients and families. Non-White older adults and individuals 
with lower education levels may particularly benefit from screening. Individuals with difficulty in any IADLs should be evaluated for possible dementia. Some available dementia screening tools, such as the GPCOG ${ }^{36}$, include informant questions regarding functional impairment. When other screening tools are used, careful assessment of functional difficulties may improve dementia detection. Older adults who attend medical visits alone for whom there is suspicion for cognitive impairment should be encouraged to bring a companion. This group may also benefit from observation for subtle signs of dementia, such as missed appointments or poorly controlled chronic conditions. Lastly, a new dementia diagnosis should be accompanied by clear communication to ensure patients and families understand the diagnosis and implications. There may be benefits of early dementia detection including safety, opportunities for future planning, and enhanced caregiver availability/support. Importantly, however, communication, counseling, and awareness are necessary to achieve any potential benefits of early diagnosis.

\section{Acknowledgements:}

Prior presentations: This paper was presented at the International Association of Gerontology and Geriatrics (IAGG) World Congress in San Francisco, California on July 25, 2017.

Corresponding Author: Halima Amjad, MD, MPH; Division of Geriatric Medicine and Gerontology Johns Hopkins University School of Medicine, Baltimore, MD, USA (e-mail: hamjad1@jhmi.edu).

Funders The National Health and Aging Trends Study (NHATS) is funded by the National Institute on Aging (UO1AG032947). Dr. Amjad received funding from the National Center for Advancing Translational Sciences/Johns Hopkins Institute for Clinical and Translational Research (KL2TR001077).

\section{Compliance with Ethical Standards:}

Conflict of Interest: Dr. Lyketsos has served as a paid consultant for Astra-Zeneca, Glaxo-Smith Kline, Eisai, Novartis, Forest, Supernus, Adlyfe, Takeda, Wyeth, Lundbeck, Merz, Lilly, Pfizer, Genentech, Elan, NFL Players Association, NFL Benefits Office, Avanir, Zinfandel, BMS, Abvie, Janssen, Orion, Otsuka, and Astellas. He has also received honoraria or travel support from Pfizer, Forest, Glaxo-Smith Kline, and Health Monitor. Drs. Amjad, Roth, Sheehan, Wolff, and Samus declare that they do not have a conflict of interest.

\section{REFERENCES}

1. Alzheimer's Association. 2017 Alzheimer's disease facts and figures. Alzheimer's \& Dementia. 2017;13(4):325-73.

2. Lang $\mathbf{L}$, Clifford $\mathbf{A}$, Wei $\mathbf{L}$, et al. Prevalence and determinants of undetected dementia in the community: A systematic literature review and a meta-analysis. BMJ Open. 2017;7(2):e011146. doi: https://doi. org/10.1136/bmjopen-2016-011146.

3. Savva GM, Arthur A. Who has undiagnosed dementia? A cross-sectional analysis of participants of the Aging, Demographics and Memory Study. Age Ageing. 2015;44(4):642-7.

4. Connolly A, Gaehl E, Martin H, Morris J, Purandare N. Underdiagnosis of dementia in primary care: Variations in the observed prevalence and comparisons to the expected prevalence. Aging Ment Health. 2011;15(8):978-84

5. Chodosh J, Petitti DB, Elliott M, et al. Physician recognition of cognitive impairment: Evaluating the need for improvement. J Am Geriatr Soc. 2004;52(7):1051-9.
6. Amjad H, Roth DL, Samus $\mathbf{Q M}$, Yasar S, Wolff JL. Potentially unsafe activities and living conditions of older adults with dementia. J Am Geriatr Soc. 2016;64(6):1223-32.

7. Borson S, Frank L, Bayley PJ, et al. Improving dementia care: The role of screening and detection of cognitive impairment. Alzheimer's \& Dementia. 2013;9(2):151-9.

8. Moyer VA, U.S. Preventive Services Task Force. Screening for cognitive impairment in older adults: U.S. Preventive Services Task Force recommendation statement. Ann Intern Med. 2014;160(11):791-7.

9. Fowler NR, Harrawood A, Frame A, et al. The Indiana University cognitive health outcomes investigation of the comparative effectiveness of dementia screening (CHOICE) study: Study protocol for a randomized controlled trial. Trials. 2014;15(1):209.

10. Wilkins CH, Wilkins KL, Meisel M, Depke M, Williams J, Edwards DF. Dementia undiagnosed in poor older adults with functional impairment J Am Geriatr Soc. 2007;55(11):1771-6.

11. Ferretti M, Seematter-Bagnoud L, Martin E, Büla CJ. New diagnoses of dementia among older patients admitted to postacute care. Journal of the American Medical Directors Association. 2010;11(5):371-6.

12. Eichler T, Thyrian JR, Hertel J, et al. Rates of formal diagnosis in people screened positive for dementia in primary care: Results of the DelpHi-trial. J Alzheimers Dis. 2014;42(2):451-8.

13. Sternberg SA, Wolfson C, Baumgarten M. Undetected dementia in community-dwelling older people: The Canadian Study of Health and Aging. J Am Geriatr Soc. 2000;48(11):1430-4.

14. NHATS data collection procedures: Round 1. 2011; Available at: http:// www.nhats.org. Accessed January 22, 2018.

15. Montaquila J, Freedman V, Edwards B, and Kasper J. National Health and Aging Trends Study round 1 sample design and selection. NHATS Technical Paper \# 1. 2012; Available at: http://www.nhats.org. Accessed January 22, 2018.

16. Kasper J, Freedman V, and Spillman B. Classification of persons by dementia status in the National Health and Aging Trends Study. NHATS Technical Paper \#5. 2013; Available at: http://www.nhats.org. Accessed January 22, 2018.

17. Galvin JE, Roe CM, Powlishta KK, et al. The AD8: A brief informant interview to detect dementia. Neurology. 2005;65(4):559-64.

18. Galvin JE, Roe CM, Xiong C, Morris JC. Validity and reliability of the AD8 informant interview in dementia. Neurology. 2006;67(11):1942-8.

19. CCW. Chronic Conditions Data Warehouse Condition Categories. Available at: https://www.ccwdata.org/web/guest/condition-categories. Accessed January 22, 2018.

20. Montaquila J, Freedman V, and Kasper J. National Health and Aging Trends Study round 1 income imputation. NHATS Technical Paper \#3. 2012; Available at: www.NHATS.org. Accessed January 22, 2018.

21. Freedman VA, Kasper JD, Cornman JC, et al. Validation of new measures of disability and functioning in the National Health and Aging Trends Study. J Gerontol A Biol Sci Med Sci. 2011;66(9):1013-21.

22. Löwe B, Kroenke K, Gräfe $\mathbf{K}$. Detecting and monitoring depression with a two-item questionnaire (PHQ-2). J Psychosom Res. 2005;58(2):163-71.

23. Kroenke K, Spitzer RL, Williams JB, Monahan PO, Löwe B. Anxiety disorders in primary care: Prevalence, impairment, comorbidity, and Detection. Ann Intern Med. 2007;146(5):317-25.

24. Charlson ME, Pompei P, Ales KL, MacKenzie CR. A new method of classifying prognostic comorbidity in longitudinal studies: Development and validation. J Chronic Dis. 1987;40(5):373-83.

25. Montaquila J, Freedman V, Spillman B, and Kasper J. National Health and Aging Trends Study development of round 1 survey weights. NHATS Technical Paper \#2. 2012; Available at: www.NHATS.org. Accessed January 22, 2018

26. Ismail Z, Smith EE, Geda Y, et al. Neuropsychiatric symptoms as early manifestations of emergent dementia: Provisional diagnostic criteria for mild behavioral impairment. Alzheimer's \& Dementia. 2016;12(2):195202.

27. Valcour VG, Masaki KH, Curb JD, Blanchette PL. The detection of dementia in the primary care setting. Arch Intern Med. 2000;160(19):2964-8.

28. Borson S, Scanlan JM, Watanabe J, Tu S, Lessig M. Improving identification of cognitive impairment in primary care. Int $\mathrm{J}$ Geriatr Psychiatry. 2006;21(4):349-55.

29. Livney MG, Clark CM, Karlawish $\mathbf{J H}$, et al. Ethnoracial differences in the clinical characteristics of Alzheimer's disease at initial presentation at an urban Alzheimer's disease center. The American Journal of Geriatric Psychiatry. 2011;19(5):430-9. 
30. Mayeda ER, Glymour MM, Quesenberry CP, Whitmer RA. Inequalities in dementia incidence between six racial and ethnic groups over 14 years. Alzheimer's \& Dementia. 2016;12(3):216-24.

31. Tang $\mathbf{M X}$, Cross $\mathbf{P}$, Andrews $\mathbf{H}$, et al. Incidence of $\mathrm{AD}$ in africanamericans, caribbean hispanics, and caucasians in northern Manhattan. Neurology. 2001;56(1):49-56.

32. Connell CM, Scott Roberts J, McLaughlin SJ. Public opinion about Alzheimer disease among blacks, hispanics, and whites: Results from a national survey. Alzheimer Dis Assoc Disord. 2007;21(3):232-40.

33. Connell CM, Scott Roberts J, McLaughlin SJ, Akinleye D. Racial differences in knowledge and beliefs about Alzheimer disease. Alzheimer Dis Assoc Disord. 2009;23(2):110-6.
34. Mahoney DF, Cloutterbuck J, Neary S, Zhan L. African american, chinese, and latino family caregivers' impressions of the onset and diagnosis of dementia: Cross-cultural similarities and differences. Gerontologist. 2005;45(6):783-92.

35. Wood RY, Giuliano KK, Bignell CU, Pritham WW. Assessing cognitive ability in research: Use of MMSE with minority populations and elderly adults with low education levels. J Gerontol Nurs. 2006;32(4):45-54.

36. Brodaty H, Pond $\mathbf{D}, \mathbf{K e m p} \mathbf{N M}$, et al. The GPCOG: A new screening test for dementia designed for general practice. J Am Geriatr Soc. 2002;50(3):530-4. 